\title{
共还原剂醛类对金属卟啉催化丙烯环氧化的作用研究
}

\author{
祁连山王涛魏永梅田恒水* \\ (华东理工大学化工学院 上海 200237)
}

\begin{abstract}
摘要 根据密度泛函理论，对分别以丙烯醛、丙醛、巴豆醛、正丁醛和异丁醛为共还原剂时，丙烯环氧化过程中可能 存在的反应路径的能垒进行了理论计算, 通过分析对比确定了最优反应路径为过氧酰基与丙烯进行自由基加成, 然后 分解为酯基自由基和环氧丙烷. 此外得到了醛类的自氧化过程以及反应过程中的脱羧反应对环氧化反应有着一定影响 的结论, 与实验数据进行对比, 二者结果比较相符. 对共还原剂醛类的篮选具有一定的指导作用.

关键词 理论计算; 丙烯环氧化; 共还原剂
\end{abstract}

\section{Study on the Effect of Co-reductant Aldehydes on Epoxidation of Propylene Catalyzed by Metalloporphyrins}

\author{
Qi, Lianshan Wang, Tao Wei, Yongmei Tian, Hengshui* \\ (School of Chemical Engineering, East China University of Science and Technology, Shanghai 200237)
}

\begin{abstract}
On the basis of density functional theory, theoretical calculation is conducted to obtain the free energy barriers of the reaction path, which may exist in the process of propylene epoxidation with co-reductant acrolein, propanal, crotonaldehyde, $N$-butyl aldehyde and isobutyl aldehyde respectively. And the optimal reaction path was determined to be free radical addition of peroxy acyl and propylene through analysis and comparison. In addition, the conclusion that auto-oxidation process of aldehydes and the decarboxylation in the reaction process have a certain influence on the epoxidation reaction can be conducted, and the results are in good consistent with the experimental data. It has a certain guiding effect on the choosing of co-reducing aldehydes.

Keywords theoretical calculation; propylene epoxidation; co-reductant
\end{abstract}

环氧丙烷(PO) 是一种非常重要的有机化工中间体, 在丙烯类衍生物中仅次于聚丙烯和丙烯腈，由于其特有 的理化性质, 被广泛应用于生产聚醚多元醇、树脂、溶 剂和表面活性剂 ${ }^{[1]}$. 目前工业上合成 PO 的生产工艺主 要是氯醇法、共氧化法(包括异丁烷共氧化法和乙苯共 氧化法)、异丙苯氧化法和过氧化氢直接氧化法 ${ }^{[2]}$. 但是 由于环保问题、工艺路线的复杂性以及原料储存的安全 隐患问题, 开发新的生产工艺迫在眉睫. 近年来有学者 通过合成特定的催化剂, 比如金负载型催化剂和钯负载 型催化剂等 ${ }^{[3 \sim 5]}$, 使用 $\mathrm{H}_{2}$ 和 $\mathrm{O}_{2}$ 在线合成 $\mathrm{H}_{2} \mathrm{O}_{2}$, 从而与 丙烯发生环氧化反应. 但是由于 PO 选择性及氢效率较 低, 该方法目前还处于实验室研究阶段 ${ }^{[6]}$. 金属卟啉能 够有效地模拟细胞色素 P-450, 具有极高的反应活性, 并且由于用量极少, 在反应后无需进行分离提纯, 避免 了分离、回收和提纯工艺所带来的高能耗. 目前金属卟
啉以及基于金属卟啉模板合成的金属有机框架材料 (MOFs) 及共轭微孔和介孔聚合物(CMPs) 材料与醛作为 共还原剂被广泛应用于烯烃环氧化中, 都表现出了优异 的催化活性和选择性 ${ }^{[7 \sim 11]}$. 因此, 金属卟啉的引入使得 氧气直接氧化丙烯成为一条合适的 PO 生产路径.

通过对比已报道的研究发现, 已有的研究主要集中 于催化剂的修饰与改性, 而对于共还原剂醛的研究较 少. 纪红兵课题组 ${ }^{[9]}$ 与我们课题组 ${ }^{[12]}$ 均发现在反应过程 中不添加醛时, 反应基本不发生, 因此共还原剂醛对于 金属卟啉催化丙烯环氧化反应有着至关重要的作用. 我 们课题组 ${ }^{[12]}$ 在 2018 年报道了以丙烯醛作为共还原剂的 丙烯环氧化过程的可能机理. 反应过程中主要的活性中 间体是丙烯醛所对应的过氧酰基自由基，其中金属卟啉 由于催化剂寿命的影响, 在整个催化反应过程中主要起 着引发反应的作用而不是催化环氧化过程. 基于上述研

* Corresponding author. E-mail: hengshuitian@126.com

Received October 23, 2019; revised December 23, 2019; published online January 21, 2020. 
究结果, 我们挑选了正丁醛、异丁醛、丙醛、巴豆醛和 丙烯醛五种小分子醛作为共还原剂, 通过理论计算与实 验相结合来进一步分析确认可能的反应机理、共还原剂 酫在反应过程中的主要反应以及对于环氧化过程的影 响, 对后续筛选共还原剂提出一定的指导建议.

\section{1 结果与讨论}

\section{1 氢转移过程能垒}

根据之前的研究结果 ${ }^{[12]}$, 在反应初期, 金属卟啉与 氧气和醛经过氧化还原反应生成酰基自由基来引发反 应, 然后酰基自由基与氧气结合生成活性中间体过氧酰 基自由基. 考虑到引发反应的速率较快以及催化剂寿命 较短，因此以活性中间体过氧酰基作为反应的起始点. 过氧酰基主要有 4 条消耗路径: (1)与金属卟啉以及丙烯 反应然后生成 $\mathrm{PO}$ 和酯基自由基; (2)分别与丙烯的 $\mathrm{C}(1)$ 和 C(2)位点进行加成反应生成中间体, 然后中间体分解 为 PO 和酯基自由基; (3)与酫反应生成过氧酸与酰基自 由基, 然后过氧酸和丙烯反应生成 PO 和酸. 其中各条 路径生成的酯基自由基再与醛发生氢转移过程生成酸 和酰基自由基，酰基自由基再与氧气结合生成过氧酰 基, 实行反应循环. 因此主要通过计算氢转移过程、过 氧酸反应路径以及自由基反应路径的自由能能垒，来对 反应机理以及最优反应路径进行分析说明.

主要的氢转移过程为酯基自由基以及过氧酰基与 醛发生氢转移反应生成酰基自由基, 然后进入下一循环 (Scheme 1).<smiles>[R]C=[O+][O-]</smiles>

图式 1 过氧酰基以及酯基与醛之间的氢转移反应

Scheme 1 Hydrogen transfer process between aldehydes and peroxyacyl radicals and ester radicals

过氧酰基和酯基与醛之间的氢转移反应的反应曲 线见图 1 和 2, 过氧酰基自由基以及酯基与其对应的醛 之间的氢转移反应过程前后的吉布斯自由能变化 $\Delta G$ 均 小于 0 , 说明上述两步反应在热力学上是可行的, 需要 从反应动力学方面分析说明反应速率差异. 过氧酰基与 醛之间的氢转移反应的自由能能垒明显高于酯基与醛 之间的氢转移反应的自由能能垒, 因而后者反应活性更 高. 并对酯基自由基和过氧酰基自由基活性位点的原子
电荷进行分析，结果如表 1 所示. 过氧酰基链端氧和酯 基单键氧的 NPA 电荷分布差距明显，在底物一样时，酯 基的夺氢能力明显高于过氧酰基，与上述计算的自由能 能垒相符. 而对于不同醛与其过氧酰基以及醛基之间能 垒的对比，趋势并不一致，原因可能在于底物不同，无 法简单的定性比较，此外在计算自由能的过程中(包括 ZPE、熵、 $\Delta H$ 校正、高精度单点能以及溶解自由能计 算)影响因素较多，而且各组之间的自由能能垒与 NPA 电荷分布差距并不明显.

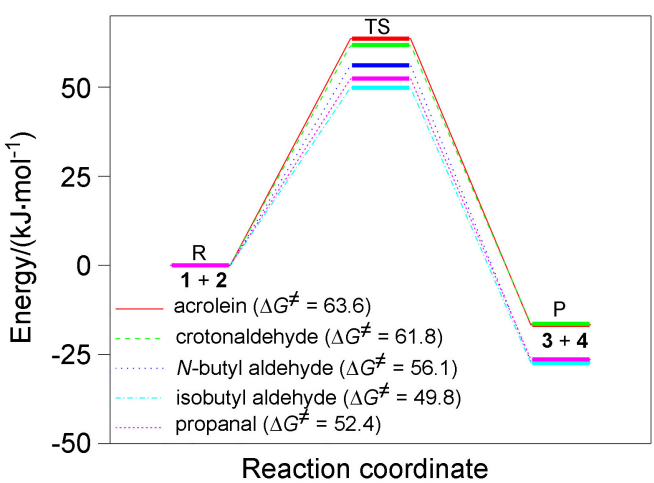

图 1 过氧酰基与醛之间的氢转移反应的反应曲线 Figure 1 Reaction profile of hydrogen transfer process between peroxyacyl radicals and aldehydes

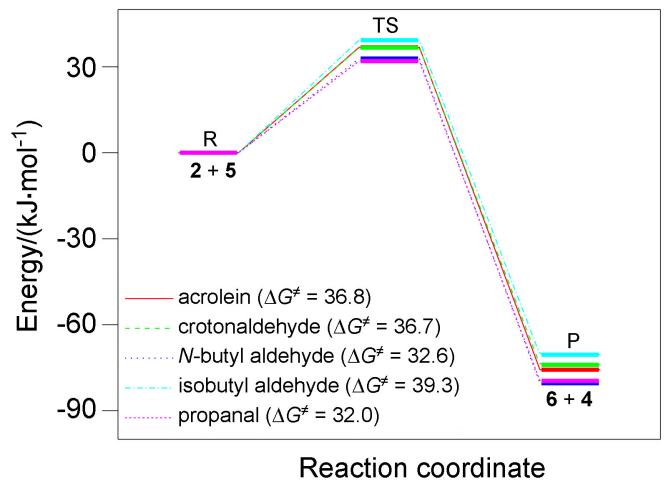

图 2 酯基与醛之间的氢转移反应的反应曲线

Figure 2 Reaction profile of hydrogen transfer process between ester radicals and aldehydes

表 1 醛、酯基、过氧酰基以及过氧酸反应位点的原子电荷 Table 1 Atomic charges of the reaction site of aldehyde, ester, peroxyacyl and peroxyacid

\begin{tabular}{lcccc}
\hline \multicolumn{1}{c}{ Aldehyde } & $\begin{array}{c}\text { Aldehyde } \\
(\mathrm{H})\end{array}$ & $\begin{array}{c}\text { Ester } \\
(\mathrm{O})\end{array}$ & $\begin{array}{c}\text { Peroxyacyl } \\
(\mathrm{O})\end{array}$ & $\begin{array}{c}\text { Peroxyacid } \\
\left(\mathrm{O}_{3}\right)\end{array}$ \\
\hline Acrolein & $0.156 e$ & $-0.419 e$ & $-0.108 e$ & $-0.447 e$ \\
Crotonaldehyde & $0.151 e$ & $-0.430 e$ & $-0.116 e$ & $-0.451 e$ \\
$N$-Butyl aldehyde & $0.149 e$ & $-0.428 e$ & $-0.109 e$ & $-0.454 e$ \\
Isobutyl aldehyde & $0.153 e$ & $-0.417 e$ & $-0.109 e$ & $-0.455 e$ \\
Propanal & $0.149 e$ & $-0.427 e$ & $-0.108 e$ & $-0.453 e$ \\
\hline
\end{tabular}

\section{2 自由基加成路径能垒}

活性中间体过氧酰基自由基与丙烯通过自由基加 
成反应生成中间体，然后分解为酯基自由基以及 $\mathrm{PO}$, 酯基自由基再与醛反应生成酰基自由基, 进入下一循 环. 根据加成位点的差别, 主要的反应路径见 Scheme 2, 根据自由基加成反应的机理，反应应该以 path 1 为主.

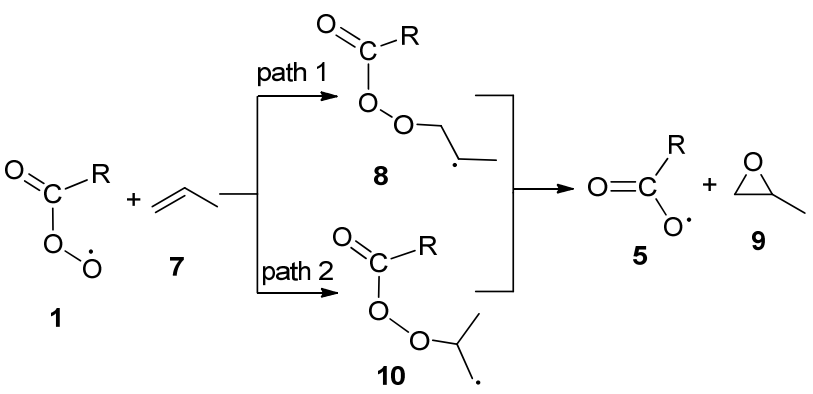

图式 2 自由基路径生成环氧丙烷

Scheme 2 PO generation path through free radical reaction

根据图 3 和图 4 对比各种醛的自由基反应路径吉布 斯自由能变化可知, 该条路径在热力学上可行, 然后从 反应动力学上定性分析各条反应路径的反应速率. 通过 对比路径中各步基元反应的自由能能垒，可知该路径的 决速步为过氧酰基与丙烯加成生成中间体. 并且对比加 成位点不同路径的自由能能垒可知, 该反应主要的加成 位点为丙烯的 $\mathrm{C}(1)$, 符合反马氏加成规则. 反应过程是 烯烃活泼的 $\pi$ 电子转移到自由基上，可以看作是亲电加 成来对比各种过氧酰基之间的加成能力. 对于巴豆醛而 言, 过氧酰基链端氧的负电荷分布较其他醛类的高, 因 此加成能力最弱, 与计算得到的能垒相符. 而对于其他 醛的 NPA 电荷分布(表 1), 差距并不明显 $(-0.108 e$ 与一 $0.109 e)$ ，与计算所得的能垒之间联系并不紧密.

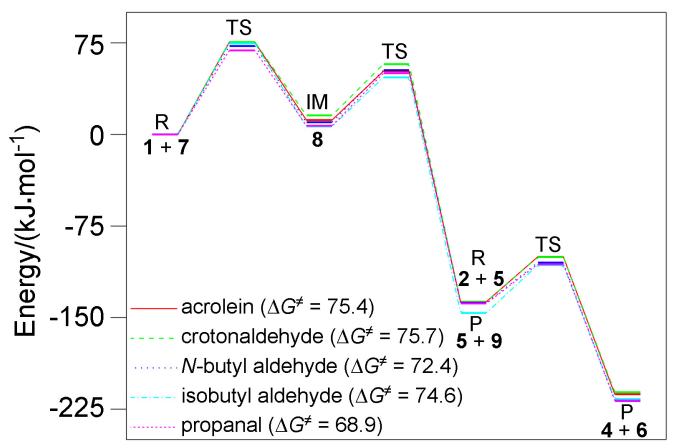

Reaction coordinate

图 3 过氧酰基与丙烯的 $\mathrm{C}(1)$ 加成反应生成环氧丙烷路径的 反应曲线

Figure 3 Reaction profile of PO generation path through free radical addition reaction between peroxyacyl radicals and $\mathrm{C}(1)$ of propylene

\section{3 过氧酸路径}

该路径主要根据 Prilezhaev Reaction 机理 ${ }^{[13]}$ 进行: 过氧酸与烯烃反应生成环氧化物以及相应的酸(Eq. 1).

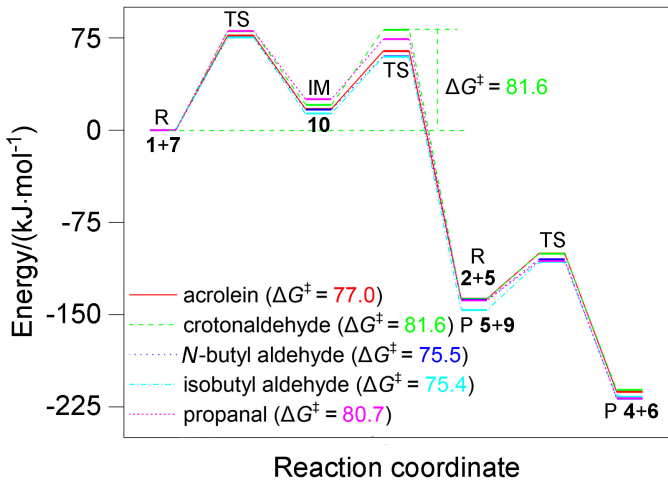

图 4 过氧酰基与丙烯的 $\mathrm{C}(2)$ 加成反应生成环氧丙烷路径的 反应曲线

Figure 4 Reaction profile of PO generation path through free radical addition reaction between peroxyacyl radical and $C(2)$ of propylene

由于本体系中通过过氧酰基与醛发生氢转移反应会生 成过氧酸，因此也对该反应路径的能垒进行了计算对 比.

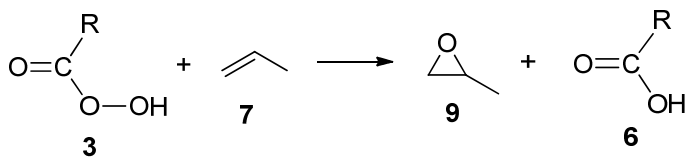

由图 5 可知, 过氧酸路径在热力学上可行. 根据各 步基元反应的自由能能垒变化，反应的决速步为环氧化 过程. 该步基元反应为协同机理，过氧酸的 $\mathrm{O}_{3}$ 接受丙烯 双键上的 $\pi$ 电子. 通过 NPA 电荷分布分析(表 1), 丙烯 醛对应的过氧酸的 $\mathrm{O}_{3}$ 的负电荷低于其他醛，更容易接 受双键上的 $\pi$ 电子，而其反应的自由能能垒最低，两者 相符较好. 而对于其他醛, 负电荷分布差距并不明显, 而计算的自由能能垒最大差距在 $1.6 \mathrm{~kJ} / \mathrm{mol}$, 也不明显, 两者之间并无明显的规律. 原因可能在于计算精度或者 溶剂的影响。

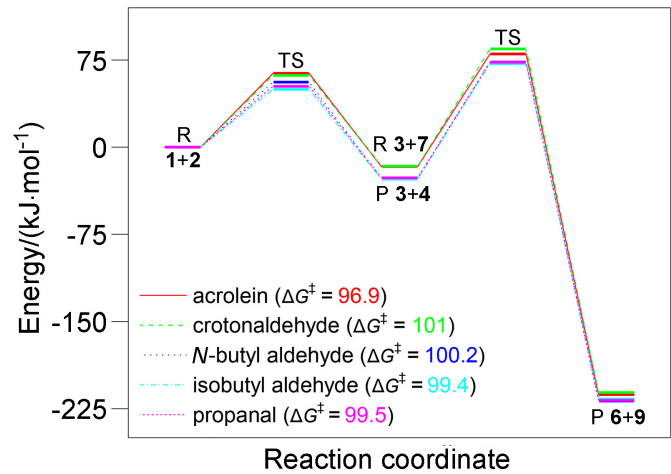

图 5 过氧酸和丙烯反应生成环氧丙烷路径的反应曲线

Figure 5 Reaction profile of PO generation path through prilezhaev reaction

上述主要的三条反应路径在热力学上均可行, 需要 从反应动力学角度来定性分析确定主要的反应路径, 主 
要通过决速步的自由能能垒来进行定性判断. 根据之前 的结果, 为了便于分析对比, 对反应过程进行简化, 以 过氧酰基自由基作为反应的起点. 过氧酸路径的自由能 能垒比相应的自由基路径高 $20 \mathrm{~kJ} / \mathrm{mol}$ 左右, 自由基路 径的反应速率明显高于过氧酸路径, 因此为本体系中的 主要反应路径, 后续活性分析中也将从反应动力学方面 考察.

\section{4 共还原剂活性考察}

在 $\mathrm{Fe}\left(p-\mathrm{CH}_{3}\right) \mathrm{TPPCl}$ 存在的情况下, 分别考察了丙 醛、丙烯醛、正丁醛、异丁醛和巴豆醛这五种共还原剂 对于反应活性的影响, 并且进行了相应的空白对照(表 2). 在存在金属卟啉时, 丙烯醛组 PO 的收率最高并且 伴随着最低的醛转化率, 然后依次是巴豆醛、丙醛、正 丁醛和异丁醛. 而与上述理论计算过程中得到的最优路 径的自由能能垒相比, 存在一定的差异, 但是却与过氧 酰基与醛之间的氢转过程能垒高低顺序相符较好. 原因 在于反应过程中活性中间体过氧酰基的消耗主要有两 类反应, 而与醛发生氢转移反应生成过氧酸的能垒较过 氧酰基与丙烯发生自由基加成的能垒低(丙烯醛 11.8 $\mathrm{kJ} / \mathrm{mol}$, 巴豆醛 $13.9 \mathrm{~kJ} / \mathrm{mol}$, 丙醛 $16.5 \mathrm{~kJ} / \mathrm{mol}$, 正丁醛 $16.3 \mathrm{~kJ} / \mathrm{mol}$, 异丁醛 $24.8 \mathrm{~kJ} / \mathrm{mol}$ ), 在相同反应条件下, 氢转移过程的反应速率较自由基加成快, 使得过氧酰基 主要生成过氧酸. 正丁醛、异丁醛和丙醛组过氧酰基以 及酯基自由基与醛之间的氢转移反应的能垒较丙烯醛 和巴豆醛组低, 这三组反应的反应速率较快, 醛基本消 耗完毕, 并且绝大部分消耗在自氧化上, 虽然其生成 PO 的能垒较低, 但是收率仍然低于高能垒的巴豆醛和 丙烯醛. 这三组的实验结果也基本与理论计算相符, 丙 醛生成 PO 的能垒最低且生成过酸的能垒较高, 从而丙 醛组的 PO 收率最高, 但是由于醛的自氧化消耗过快, 导致三者的差距并不明显.

对于丙烯醛和巴豆醛, 虽然其生成 PO 的能垒较高, 导致其反应速率较低, 但是其自氧化过程的能垒较高, 并且两者决速步的自由能能垒差距相对较小, 两者的反 应速率差距较其他醛类低, 使得醛的消耗更偏向于环氧 化过程, 二者反应结果与计算结果相符较好. 因此醛的 自氧化与环氧化的竞争对于反应活性有着重要的影响. 后续篮选共还原剂时，应该综合考虑醛的自氧化和环氧 化能力，而且金属卟啉的引入，也避免了不活泼醛难以 引发反应导致诱导期过长的问题.

此外，过氧酸在高温以及金属离子存在的情况下， 会发生过氧键断裂生成酯基和羟基自由基或者氢氧根 离子 ${ }^{[14,15]}$. 由金属卟啉组以及空白组的对比可得, 金属 卟啉组的 PO 收率均要低于空白组, 并且酸的选择性也 要低于空白组. 原因在于该反应体系中金属卟啉主
表 2 共还原剂活性考察 ${ }^{a}$

Table 2 Study on the activity of Co-reductant

\begin{tabular}{ccccc}
\hline Entry & Aldehyde & Yield/\% & Conversion/\% & Selectivity/\% \\
\hline 1 & \multirow{2}{*}{ Acrolein } & 30.0 & 68.0 & 37.2 \\
$1^{b}$ & & $-^{c}$ & - & - \\
2 & \multirow{2}{*}{ Crotonaldehyde } & 17.9 & 88.1 & 36.7 \\
$2^{b}$ & 21.8 & 86.4 & 39.6 \\
3 & \multirow{2}{*}{ Propanal } & 14.6 & 97.7 & 66.0 \\
$3^{b}$ & & 17.5 & 97.7 & 70.9 \\
4 & \multirow{2}{*}{ N-Butyl aldehyde } & 12.8 & $>99.0$ & 71.0 \\
$4^{b}$ & & 14.4 & $>99.0$ & 72.7 \\
5 & \multirow{2}{*}{ Isobutyl aldehyde } & 10.7 & $>99.0$ & 42.2 \\
$5^{b}$ & & 12.3 & $>99.0$ & 43.8 \\
\hline
\end{tabular}

${ }^{a}$ Reaction conditions: propylene $(10 \mathrm{~L})$, bubbling rate $(0.1 \mathrm{~L} / \mathrm{min})$, molar ratio of aldehyde/propylene is $1: 1,100{ }^{\circ} \mathrm{C}$, ethyl acetate $(200 \mathrm{~mL})$, stirring speed $(500 \mathrm{r} / \mathrm{min}), \quad \mathrm{Fe}\left(p-\mathrm{CH}_{3}\right) \mathrm{TPPCl}\left(1.6 \times 10^{-5} \mathrm{~mol} / \mathrm{L}\right), 1 \mathrm{~h} .{ }^{b}$ Without $\mathrm{Fe}\left(p-\mathrm{CH}_{3}\right) \mathrm{TPPCl}^{c}{ }^{c}$ No reaction

要作为引发剂, 然后会发生分解产生金属离子. 而金属 离子对于过氧酸的分解有着一定的促进作用，使得酯基 以及羟基自由基的含量较空白组多，从而导致了酯基脱 去二氧化碳的反应速率增加, 最终导致酸的选择性出现 了小幅下降. 而对于异丁醛组酸的选择性明显低于正丁 醛以及丙醛, 原因在于中间体分解为 PO 和酯基的过程 中, 酯基会发生均裂生成异丙基自由基与二氧化碳，该 过程在计算机模拟过程中有着很好的体现. 而对于丙烯 醛和巴豆醛组酸选择性较低的原因在于两者对应的醛 和酸在反应体系内会发生一定程度的聚合.

最后，对于共还原剂醛的副反应，由于过氧酸的均 裂产生的羟基自由基与脱羧过程中产生的烷基自由基 以及乙烯基或丙烯基自由基结合生成相应的醇或者烯 醇，然后进一步氧化生成酸或者酮，因此反应体系中应 该存在着相应的副产物: 丙烯醛组副产物为乙酸, 丙醛 组副产物为乙酸和乙醇, 正丁醛组为丙酸和正丙醇, 异 丁醛组为异丙醇和丙酮, 巴豆醛组为乙酸和丙酸, 该结 论与实验结果相符.

\section{2 结论}

通过计算多种共还原剂体系中可能存在的反应路 径的能垒, 进一步确定了活性中间体为过氧酰基. 在一 定程度上阐述了共还原剂对于体系的影响方式, 自氧化 过程与环氧化过程的竞争反应影响着环氧丙烷的收率 对于后续工作中共还原剂的節选有着一定的指导作用. 此外, 对反应体系中可能的副反应，例如过氧酸以及过 氧酰基等的脱䊣也做了一定的分析说明.

\section{3 实验部分}

\section{1 仪器与试剂}

紫外可见光谱通过紫外可见分光光度计 Varian 
CARY 100 得到, 红外光谱通过傅里叶红外光谱仪 Nicolet6700 得到, 主副产物定性通过气相色谱一质谱联 用仪 Agilent 6890-5973N 分析得到. 所用试剂与药品均 为市售的分析纯试剂, 吡咯经重新蒸馏提纯, 所合成的 卟啉通过柱色谱提纯. 理论计算部分采用 Gaussian $09^{[16]}$ 软件, 计算过程在华东理工大学高性能计算中心 进行.

\section{2 实验方法}

催化剂合成按照文献[17]进行合成, 表征数据与文 献相符.

共还原剂活性考察: 在反应釜内加入 $200 \mathrm{~mL}$ 乙酸 乙酯、金属卟啉 $\left(1.6 \times 10^{-5} \mathrm{~mol} / \mathrm{L}\right)$ 以及一定量的醛 $($ 醛与 丙烯的物质的量之比为 $1: 1$ )进行摚拌, 再通入 $10 \mathrm{~L}$ 丙 烯, 加热到 $100{ }^{\circ} \mathrm{C}$, 然后按照 $0.1 \mathrm{~L} / \mathrm{min}$ 的速度通入氧 气，反应时长为 $1 \mathrm{~h}$, 反应完成后进行冷却取料分析.

理论计算部分：所有的理论计算部分在 Gaussian 09 程序包下完成, 在 B3LYP/6-31G(d,p $)^{[18,19]}$ 下进行结构 优化以及频率分析, 过渡态通过 TS 方法搜索, 并且通 过唯一虚频以及内禀反应坐标(IRC)来进行验证, 以上 步骤均在气相状态下进行, 并在隐式溶剂模型下计算 NPA 电荷 ${ }^{[20]}$ 分布. 然后在双杂化泛函 B2PLYPD $3^{[21]}$ 以及 def2-TZVPP ${ }^{[22]}$ 基组下计算电子能量, 溶解自由能通过 隐式溶剂模型下计算得到的单点能减去气相下计算得 到的单点能, 计算方法为 $M 05-2 X / 6-31 G(d)^{[23,24]}$, 并通 过频率校正因子 ${ }^{[25]}$ 得到热力学校正量, 最终得到溶质 在溶剂环境下的自由能，计算公式如下：

$$
G_{\text {sion }}=G_{6-31 \mathrm{G}(\mathrm{d}, \mathrm{p})}+E_{\text {sion }}-E_{\text {gas }}+\mathrm{ZPE}+\Delta H-\mathrm{TS}+10.6
$$

辅助材料(Supporting Information) 过渡态结构的 IRC 验证图. 这些材料可以免费从本刊网站 (http://siocjournal.cn/)上下载.

\section{References}

[1] Nijhuis, T. A.; Makkee, M.; Moulijn, J. A.; Weckhuysen, B. M. Ind. Eng. Chem. Res. 2006, 45, 3447.

[2] Russo, V.; Tesser, R.; Santacesaria, E.; Di Serio, M. Ind. Eng.
Chem. Res. 2013, 52, 1168

[3] Chowdhury, B.; Bravo-Suárez, J. J.; Daté, M.; Tsubota, S.; Haruta, M. Angew. Chem., Int. Ed. 2006, 45, 412.

[4] Du, M.; Huang, J.; Sun, D.; Li, Q. Appl. Surf. Sci. 2016, 366, 292.

[5] Hikazudani, S.; Mochida, T.; Yano, K.; Nagaoka, K.; Ishihara, T.; Takita, Y. Catal. Commun. 2011, 12, 1396.

[6] Chen, J.; Halin, S. J. A.; Schouten, J. C.; Nijhuis, T. A. Faraday Discuss. 2011, 152, 321.

[7] Sun, C.; Hu, B.; Liu, Z. Chem. Eng. J. 2013, 232, 96

[8] Zhou, X.; Ji, H. Chem. Eng. J. 2010, 156, 411.

[9] Li, Y.; Zhou, X.; Chen, S.; Luo, R.; Jiang, J.; Liang, Z.; Ji, H. RSC Adv. 2015, 5, 30014.

[10] Brown, J. W.; Nguyen, Q. T.; Otto, T.; Jarenwattananon, N. N.; Glöggler, S.; Bouchard, L. S. Catal. Commun. 2015, 59, 50.

[11] Chen, L.; Yang, Y.; Guo, Z.; Jiang, D. Adv. Mater. 2011, 23, 3149

[12] Qi, L.; Wang, T.; Wei, Y.; Tian, H. Eur. J. Org. Chem. 2018, 2018, 6557.

[13] Koerner, T.; Slebocka-Tilk, H.; Brown, R. S. J. Org. Chem. 1999, 64, 196.

[14] Bawn, C. E. H.; Williamson, J. B. Trans. Faraday Soc. 1951, 47, 735.

[15] Bawn, C. E. H.; Hobin, T. P.; Raphael, L. Proc. R. Soc. A Math Phys. Eng. Sci. 1956, 237, 313.

[16] Frisch, M. J.; Trucks, G. W.; Schlegel, H. B.; Scuseria, G. E.; Robb, M. A.; Cheeseman, J. R.; Scalmani, G.; Barone, V.; Mennucci, B.; Petersson, G. A.; Nakatsuji, H.; Caricato, M.; Li, X.; Hratchian, H. P.; Izmaylov, A. F.; Bloino, J.; Zheng, G.; Sonnenberg, J. L.; Hada, M.; Ehara, M.; Toyota, K.; Fukuda, R.; Hasegawa, J.; Ishida, M.; Nakajima, T.; Honda, Y.; Kitao, O.; Nakai, H.; Vreven, T.; Montgomery, J. A. Jr.; Peralta, J. E.; Ogliaro, F.; Bearpark, M.; Heyd, J. J.; Brothers, E.; Kudin, K. N.; Staroverov, V. N.; Keith, T.; Kobayashi, R.; Normand, J.; Raghavachari, K.; Rendell, A.; Burant, J. C.; Iyengar, S. S.; Tomasi, J.; Cossi, M.; Rega, N.; Millam, J. M.; Klene, M.; Knox, J. E.; Cross, J. B.; Bakken, V.; Adamo, C.; Jaramillo, J.; Gomperts, R.; Stratmann, R. E.; Yazyev, O.; Austin, A. J.; Cammi, R.; Pomelli, C.; Ochterski, J. W.; Martin, R. L.; Morokuma, K.; Zakrzewski, V. G.; Voth, G. A.; Salvador, P.; Dannenberg, J. J.; Dapprich, S.; Daniels, A. D.; Farkas, O.; Foresman, J. B.; Ortiz, J. V.; Cioslowski, J.; Fox, D. J. Gaussian 09, Revision C.01, Gaussian, Inc., Wallingford CT, 2010.

[17] Sun, Z.; She, Y.; Zhong, R. Front. Chem. Eng. China 2009, 3, 457.

[18] Becke, A. D. J. Chem. Phys. 1993, 98, 5648.

[19] Lee, C.; Yang, W.; Parr, R. G. Phys. Rev. B 1988, 37, 785 .

[20] Reed, A. E.; Weinstock, R. B.; Weinhold, F. J. Chem. Phys. 1985, 83,735

[21] Grimme, S.; Antony, J.; Ehrlich, S.; Krieg, H. J. Chem. Phys. 2010, 132,154104

[22] Weigend, F.; Ahlrichs, R.; Gmbh, F. K. Phys. Chem. Chem. Phys. 2005, 7, 3297

[23] Marenich, A. V.; Cramer, C. J.; Truhlar, D. G. J. Phys. Chem. B 2009, 113, 6378

[24] Ho, J.; Klamt, A.; Coote, M. L. J. Phys. Chem. A 2010, 114, 13442.

[25] Merrick, J. P.; Moran, D.; Radom, L. J. Phys. Chem. A 2007, 111, 11683. 